\title{
Incoherent exciton echo on the CdSe/CdS/ZnS semiconductor quantum dots
}

\author{
Vitaly Samartsev ${ }^{1, *}$, Tatiana Mitrofanova ${ }^{1}$, and Alexander Saiko ${ }^{2}$ \\ ${ }^{1}$ FRC Kazan Scientific Center of Russian Academy of Sciences, 420029 Kazan, Russia \\ ${ }^{2}$ Scientific and Production Center of the National Academy of Sciences of Belarus for Materials \\ Science, 220072 Minsk, Belarus
}

\begin{abstract}
The possibility and conditions of an incoherent exciton echo excitation in a thin layer of the $\mathrm{CdSe} / \mathrm{CdS} / \mathrm{ZnS}$ semiconductor quantum dots spread on a glass substrate are discussed.
\end{abstract}

An experiment on the incoherent photon echo (IPE) in a thin layer of $\mathrm{CdSe} / \mathrm{CdS} / \mathrm{ZnS}$ semiconductor quantum dots (QDs) spread on a glass substrate was reported in [1-3]. The authors studied QDs manufactured by «QD-light» (Russia) using the colloidal synthesis technique. The size of the $\mathrm{CdSe} / \mathrm{CdS} / \mathrm{ZnS}$ QDs was 3-7 $\mathrm{nm}$. It should be noted that the presence of two shells of $\mathrm{CdS}$ and $\mathrm{ZnS}$ around the CdSe core contributes to an improvement in the emissivity, photostability, and high quantum yield compared to nanocrystals with a single shell and without a shell. It was shown that $\mathrm{CdSe} / \mathrm{CdS} / \mathrm{ZnS}$ QDs are uniformly distributed over a thin film layer, and the excitation should be carried out at a wavelength of $580 \mathrm{~nm}$ and a film temperature of $10 \mathrm{~K}$, when the transverse irreversible relaxation time $T_{2}$ is equal to $0.75 \mathrm{ps}$.

In the study [1], the experimental technique of IPE was used, which was described in detail in [4-6] and in the monograph [7]. The experimental work [8] (and the monograph by Novotny and Hecht [9]) states that a photon excitation of semiconductor QDs necessarily leads to an excitation of Wannier-Mott excitons, whose size is approximately equal to the size of QDs. The question arises: was the excitation of Wannier-Mott excitons taken into account in [1-3] and was there an incoherent exciton echo (IEE) at localized excitons?

Note that the excitation of laser pulses and the capture of optical responses is conveniently carried out using prisms of total internal reflection [10]. In the IPE mode, a noise optical pulse will first excite free electron-hole pairs [11], which then, losing energy, turn into coupled electron-hole pairs, i.e. to Wannier-Mott excitons, which are the only form of a photon excitation in semiconductor QDs. Following [12], we assume that these excitons will be localized, and the experimentally observed response [1-3] is the IEE at local excitons.

In Russia, the IPE technique was implemented by Yu.G. Vainer [4], who rightly asserted that the temporal resolution of the photon echo method is determined in the general case not by the duration of the exciting pulses $\Delta t$, but by the radiation coherence time $\tau_{\mathrm{c}}$, i.e. the reciprocal of the spectrum width of these pulses. Then, when using a broadband optical

\footnotetext{
* Corresponding author: samartsev@kfti.knc.ru
} 
source $\left(\tau_{\mathrm{c}} \ll \Delta t\right)$, the temporal resolution of the photon echo method can reach tens of femtoseconds (see [4, 13]). Such pulsed sources (with a spectrum width of hundreds of $\mathrm{cm}^{-1}$ ) are called noise sources. The dye laser with $\Delta t=15 \mathrm{~ns}$ can serve as such source.

The most adequate model of the IPE formation is the model of accumulated stimulated photon echo (ASPE), described in detail in $[4,13]$. In fact, the method of IPE excitation in a thin layer of semiconductor QDs is similar to the method of IEE excitation, especially when it is excited on localized Wannier-Mott excitons. The value of optical dephasing time $T_{2}$ obtained in [1-3] was $750 \mathrm{fs}$, and the homogeneous linewidth of the transition $1 / \pi T_{2}$ was equal to $424.63 \mathrm{GHz}$ at a temperature $T=10 \mathrm{~K}$. Thus, in the studied thin layer of $\mathrm{CdSe} / \mathrm{CdS} / \mathrm{ZnS}$ QDs, an unexpectedly large shortening of the optical dephasing time was found, which was attributed to a number of reasons. But the reason associated with the excitation of Wannier-Mott excitons was not mentioned. It is known [14] that an excitonecho is formed only in the case of non-bosonic commutation relations between the creation and annihilation operators of excitons, i.e. IEE will be absent in the case of small "areas" of the exciting pulses, while the IPE will still be formed. We suppose that in the experiment [1-3], the localized Wannier-Mott excitons were formed, since in the case of free excitons, the optical dephasing time obtained from the analysis of the time decay of the IEE signal would be, according to [12], 5 fs. In our opinion, we are talking about localization of excitons on the $\mathrm{CdS}$ and $\mathrm{ZnS}$ shells.

The reported study was funded by RFBR and BRFBR, project number 18-52-00026.

\section{References}

1. K.R. Karimullin, M.V. Knyazev, A.I. Arzhanov, L.A. Nurtdinova, A.V. Naumov, J. Phys. Conf. Ser. 859, 012010 (2018)

2. A.I. Arzhanov, K.R. Karimullin, A.V. Naumov, Bull. Lebedev Phys. Inst. 45, 91 (2018)

3. K.R. Karimullin, A.I. Arzhanov, A.V. Naumov, Bull. Rus. Acad. Sci. Phys. 82, 1620 (2018)

4. Y.G. Vainer, N.V. Gruzdev, Opt. Spectrosc. 76, 236 (1994)

5. M.V. Knyazev, K.R. Karimullin, A.V. Naumov, Phys. Stat. Sol. (RRL). 11, 1600414 (2017)

6. K. Karimullin, M. Knyazev, I. Eremchev, Y. Vainer, A. Naumov, Meas. Sci. Technology. 24, 027002 (2013)

7. V.V. Samartsev, V.G. Nikiforov, Femtosecond laser spectroscopy (Trovant, Moscow, 2017)

8. X. Li, Y. Wu, D. Steel et al., Science 301, 809 (2003)

9. L. Novotny, B. Hecht, Principles of nano-optics (Cambridge University Press, Cambridge, 2006)

10. R.A. Vlasov, O.N. Gadomskii, I.V. Gadomskaya, V.V. Samartsev, Zh. Eksp. Teor. Fiz. 90, 1938 (1986)

11. I.S. Osad'ko, Fluctuating fluorescence of nanoparticles (Fizmatlit, Moscow, 2011)

12. G. Noll, W. Siegner, S.G. Shevel, E.O. Gobel, JETP Lett. 51, 409 (1990)

13. V.V. Samartsev, A.M. Shegeda, A.V. Shkalikov, K.R. Karimullin, T.G. Mitrofanova, V.A. Zuikov, Las. Phys. Lett. 4, 534 (2007)

14. V.V. Samartsev, Phys. Lett. A. 38, 363 (1972) 